\title{
A novel genetic- and cell-based tool for assessing the efficacy and toxicity of anticancer drugs in vitro
}

\author{
Lan Kluwe', Wei Jiang ${ }^{\mathrm{b}}$, Ina Alster ${ }^{\mathrm{b}}$, Henning Hanken ${ }^{\mathrm{a}}$
}

\begin{abstract}
Aims. To develop an in vitro tool for assessing the efficacy and toxicity of anticancer drugs using mixed culture containing both tumor and non-tumor cells. Such in vitro tool should have high application potential in drug-screening and personalized cancer care.

Methods. Fibroblasts were spiked as non-tumor cells into tumor cells of an established line. The mixed culture was treated with a test drug at various concentrations. After the treatment, DNA was prepared directly from the survived adhesive cells in the wells of the 96-well plates using a simple and inexpensive method, and subjected to digital PCR for measuring relative copy numbers of a target gene NF1 to that of a reference gene RPP30. The NF1 gene is known to be heterozygously deleted in these tumor cells while the RPP30 gene has two copies in both tumor and non-tumor cells. Using the NF1/ RPP30 ratios resulting from the dual digital PCR assay, the proportions of tumor cells were calculated for each drug concentration.

Results. Digital PCR confirmed that the tumor cells have only one copy of the NF1 gene while the non-tumor fibroblasts have two copies. By contrast, both types of cells have two copies of the reference gene RPP30. Using the ratio of the two genes, we successfully calculated the proportion of tumor cells which decreased as the dose of the test drug increased up to a certain concentration, indicating that the drug is more effective for the tumor cells than for the non-tumor cells in this dose-range. At the highest dose, we observed a slight increase in the proportion of tumor cells, likely reflecting the toxic effect of the drug on both tumor and non-tumor cells.

Conclusion. This pilot study demonstrated the feasibility of a genetic- and cell-based tool for testing efficacy and toxicity of anticancer drugs in vitro. The promising results suggest that additional efforts are merited, for further development since such a tool will likely have high application potential (1) in drug discovery where it enables simultaneously assessing therapeutic effect on target cells and toxic effect on non-target cells, and (2) in personalized adjuvant chemotherapy where multiple drugs can be tested in primary cultures derived from surgically removed tumor.
\end{abstract}

Key words: drug discovery; drug specificity; digital PCR; primary culture; mixed culture; chemotherapy; drug selection; mutation; copy number variation

Received: April 21, 2015; Accepted with revision: November 4, 2015; Available online: November 10, 2015 http://dx.doi.org/10.5507/bp.2015.057

aLaboratory for Tumor Biology and Regenerative Medicine, Department of Oral and Maxillofacial Surgery, University Medical Center Hamburg-Eppendorf, Hamburg, Germany

${ }^{b}$ Department of Neurology, University Medical Center Hamburg-Eppendorf, Hamburg, Germany

Corresponding author: Lan Kluwe, e-mail: kluwe@uke.de

\section{INTRODUCTION}

Like medical drugs in general, the desired therapeutic effect of an anticancer drug is usually associated with some undesired toxicity, which is an essential issue in drug discovery as well as in treatment of patients. The therapeutic index (or therapeutic window), the distance between the doses at each of which the target cells and the non-target cells are affected to similar extent, reflect the specificity of the $\operatorname{drug}^{1}$ (Fig. 1). At present, the therapeutic index is difficult to assess ex vivo, largely due to the lack of suitable tools. Cell cultures containing both tumor and non-tumor cells would provide a potential in vitro resource for investigating the therapeutic index since the desired therapeutic effect on tumor cells and toxic effects on non-tumor cells can be followed simultaneously under identical experimental conditions. However, conventional in vitro assays such as proliferation and viability assays, measure the efficacy of a drug on all cells. They cannot divide the efficacy into the part on tumor cells and that on non-tumor cells. For example, a 50\% reduction in total cell number of a culture composed of $50 \%$ tumor cells and $50 \%$ non-tumor cells can imply death of all tumor cells, death of all non-tumor cells or, more likely, death of both in varying ratios.

Also because of the technical obstacle in using cultures composed of heterogeneous cells, primary cultures are only rarely suitable for testing drugs. On the other hand, owing to the cellular heterogeneity, primary cultures resemble their original tumors more than clonal cells in established cell lines ${ }^{2}$. More importantly, primary cultures are patient-specific and therefore offer the potential for individualized drug testing ${ }^{3}$.

A tool for determining the proportion of tumor cells in mixed cultures and/or in primary cultures is therefore highly desirable. Toward this challenge, we conceived a novel genetic-based strategy. A tumor is the result of somatic genetic alterations; the tumor cells exclusively have 


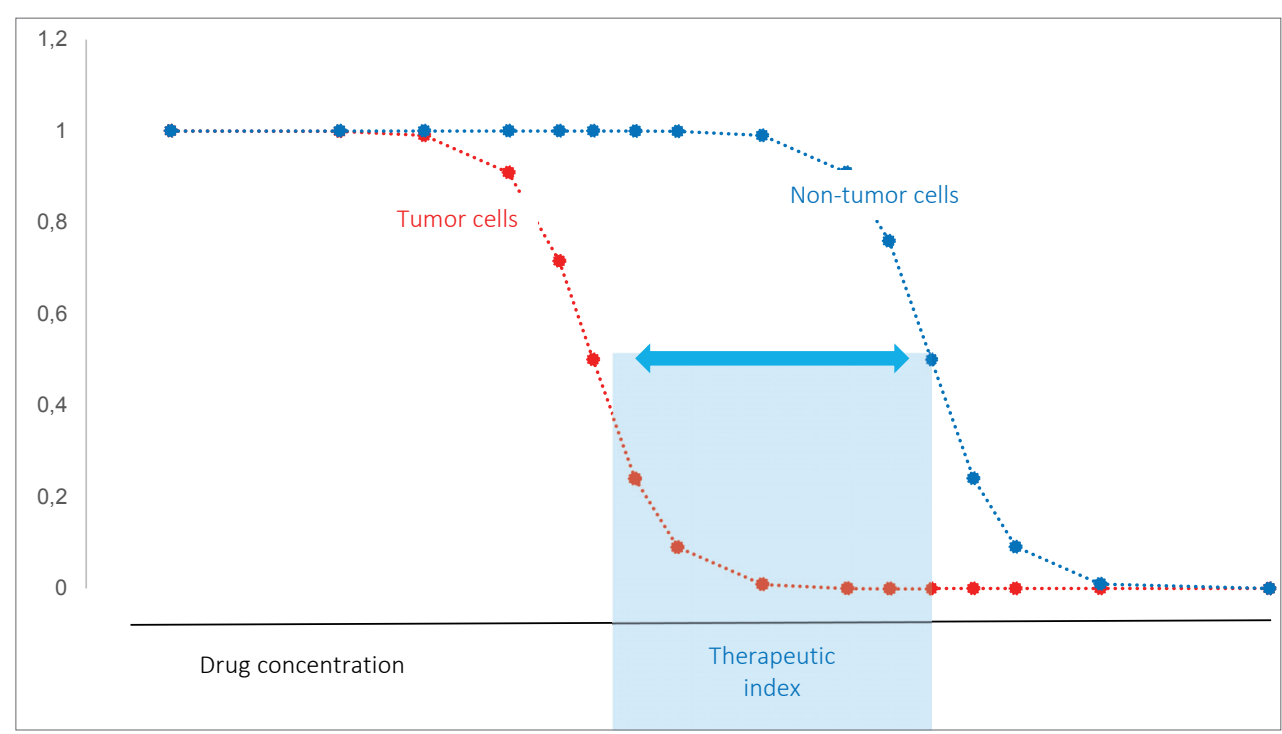

Fig. 1. Therapeutic index (blue area), the difference between two doses: one for $50 \%$ maximum effect on tumor cells and the other for $50 \%$ maximum effect on non-tumor cells.

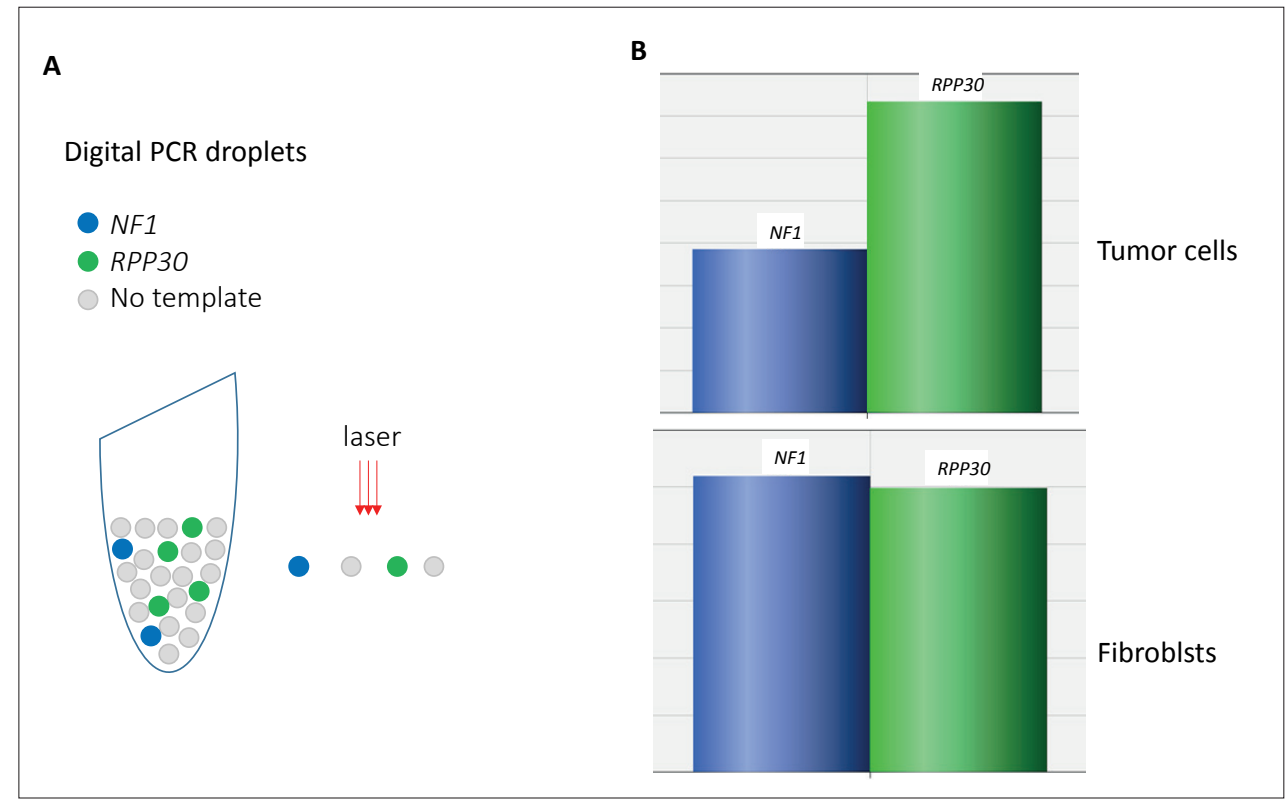

Fig. 2. Digital PCR. Illustration of the principle.

(A) and the results of a dual assay revealing relative amounts of a target (NF1) gene and a reference ( RPP30) gene (B). In the tumor cells, the ratio of NF1: RPP30 is $0.5=1 / 2$ while in the non-tumor fibroblasts this ratio is $1.0=2 / 2$. In a culture containing tumor and non-tumor cells at a ratio of $50 \%: 50 \%$, the ratio of $N F 1$ : RPP30 should be $0.75=1.5 / 2$. Consequently, the proportion of tumor cells in a mixed culture can be calculated from the ratio of NF1: RPP30 which can be determined with digital PCR.

mutations and/or copy number variations, which are not present in non-tumor cells. By quantifying one or more such genetic alterations in relation to a universal sequence which is present as two copies in both tumor and non-tumor cells, the proportion of tumor cells in a mixed culture can be derived. With rapid progress in genetic technologies, precise quantification of a sequence and a genetic alteration can be easily and quickly achieved today. Digital PCR is one such technology, which simultaneously quantifies a target sequence and a universal reference sequence in one reaction (Fig. 2A).
In this pilot study, we implemented this genetic and cell-based strategy for assessing the efficacy and toxicity of anticancer drugs. We spiked fibroblasts as non-tumor cells into tumor cells of an established malignant cell line, treated the mixed culture with a test drug, quantified a tumor-specific genetic alteration and calculated the proportion of tumor cells at each drug concentration. 


\section{MATERIALS AND METHODS}

\section{Preparation of mixed culture of tumor and non-tumor cells}

Tumor cells were from an established cell line (S462) derived from malignant nerve sheath tumor. These tumor cells were genetically verified to have a heterozygous allelic loss of the tumor suppressor gene $N F 1$ (ref. ${ }^{4}$ ).

Fibroblasts were derived from the skin of a subcutaneous fat tissue specimen of a healthy donor who underwent a resection for a medical indication and donated the disposed specimen for another study, which had been approved by the local medical review board (WF-015/12). The patient was informed about the study and gave written informed consent. The specimen was anonymized.

Both the tumor S462 cells and the non-tumor fibroblasts were cultured in standard medium under standard cultural conditions, and harvested using trypsin ${ }^{5}$.

For validating the method, tumor cells and non-tumor cells were mixed in various ratios (Table 1) and plated into wells of a 96-well plate at density of 4000 cells/well. Each ratio was set up in 4 replicates. The cells were left overnight for attachment. On the next day, the medium was aspirated and the attached cells were washed once with PBS and subjected to DNA preparation.

For creating mixed culture for the treatment, 400 cells of the S462 cell line and 1600 fibroblasts were added to each well of a 96-well culture plate, giving a density of 2000 cells/well with a tumor and non-tumor ratio of 1:4. On the next day, the medium was changed with that containing the test drug at various concentrations including a control with no drug. The treatment was continued for 5 days.

At the end of the treatment, cells were photo-documented. Subsequently, the medium was aspirated and the surviving adhesive cells were washed once with PBS and subjected to DNA preparation.

\section{DNA preparation}

DNA for digital PCR was prepared (not purified) directly from living cells attached on the culturing surface of a 96-plate using a simple, quick and inexpensive method which includes an initial cell lysis by $\operatorname{HotShot}^{6}$ and subsequent desalting by Drop-Dialysis ${ }^{7}$.

\section{HotShot: lysis of cells}

- $\quad 50 \mu \mathrm{L}$ alkaline lysis solution $(25 \mathrm{mM} \mathrm{NaOH}, 0.2 \mathrm{mM}$ EDTA) was added to each well of a 96-well culture plate

- Wells were sealed with foil to prevent evaporation and the plate was incubated at $95^{\circ} \mathrm{C}$ in an oven or on a heating plate for $30 \mathrm{~min}$ to lyse the cells

- $50 \mu \mathrm{L}$ neutralizing solution (40mM Tris-HC, $\mathrm{pH} 4.0$ ) was added to each well.

- The lysis suspension was dried in a PCR cycler at $95^{\circ} \mathrm{C}$ for 10 to $30 \mathrm{~min}$ and subsequently dissolved in $20 \mu \mathrm{L}$ distilled water

- The culture-plate was checked under microscope to ensure complete lysis

\section{Drop-Dialysis: remove salts and other contaminants}

- A membrane filter of $25 \mathrm{~mm}$ diameter (type-VS Millipore membrane with mean pore size of 0.025 $\mu \mathrm{m})$ was divided into 4 numbered areas with a pen.

- The filter was floated on distilled water with shiny side up in wells of a 24-well plate and wetted for 5 min. Attention was paid to ensure no air bubbles were trapped between the filter and water.

- The $20 \mu \mathrm{L}$ lysis solution containing DNA was pipetted carefully onto on each area of the filter. The 24-well plate was covered and dialyzed for 30 to $60 \mathrm{~min}$. The drop may suck in water due to osmotic effect.

- Water under the filter was carefully aspirated without flipping over the filter or allowing water to flow onto the filter

- DNA-drops were easily transferred into wells of a PCR-plate and

- Dried by heating $95{ }^{\circ} \mathrm{C}$ for 10 to $30 \mathrm{~min}$ in a PCR cycler

- DNA was dissolved in $10 \mu \mathrm{L}$ water and subjected to digital PCR

\section{Digital PCR}

Two validated ddPCR copy number assays, one for the NF1 gene labelled with FAM (dHsaCP1000177, 17: 29654623-29654745) and the other for the RPP30 reference gene labelled with HEX (dHsaCP2500350, ch10: 92660373-92660495) were purchased from BioRad. Also other components for the assays including droplet PCR supermix, cartridge, gaskets and foil seals were also purchased from BioRad. HaeIII was purchased from New

Table 1. Proportion of tumor cells: set-up and calculated.

\begin{tabular}{cccc}
\hline \multirow{2}{*}{$\begin{array}{c}\text { Numbers of cells / well } \\
\text { Tumor cells }\end{array}$} & Fibroblasts & Set up & \begin{tabular}{c} 
Proportion of tumor cells \\
\cline { 2 - 4 }
\end{tabular} \\
\hline 0 & 4000 & 0.00 & $\begin{array}{c}\text { Calculated from measured ratio of NF1/RPP30 } \\
\text { (mean } \pm \text { standard deviation) }\end{array}$ \\
670 & 3300 & 0.17 & $0.00 \pm 0.08$ \\
1300 & 2700 & 0.33 & $0.37 \pm 0.09$ \\
2000 & 2000 & 0.50 & $0.56 \pm 0.12$ \\
2700 & 1300 & 0.65 & $0.67 \pm 0.22$ \\
3300 & 670 & 0.83 & $0.75 \pm 0.09$ \\
4000 & 0 & 1.00 & $0.90 \pm 0.05$ \\
\end{tabular}


England Lab. The setting up and reaction of digital PCR was as described in the protocol provided by BioRad.

\section{Calculating proportion of tumor cells}

The raw data of digital PCR were analyzed automatically by an integrated program, which gave the number of positive and negative droplets and ratio of target and reference which was NF1/ RPP30 in this study. For each reaction, the events of positive and negative droplets were controlled manually. Those not meeting the criteria were excluded from further analysis. For reactions where numbers of droplets were reasonable but automatic analysis failed, thresholds for positive and negative droplets were reset manually, which enabled the analysis. The resulting ratios of NF1/ RPP30 were exported to an excel data sheet which was used to calculate the proportion of tumor cells in a mixed culture as:

Number of tumor cells / number of total cells $=2$ * (1- NF1/ RPP30)

This equation was derived as following:

- Number of NF1 = number of tumor cells $+2 *$ number of non-tumor cells

- Number of RPP30 $=2$ * number of tumor cells +2 * number of non-tumor cells

\section{RESULTS}

Using DNA prepared by the combined HotShot/DropDialysis method, we successfully obtained reasonable results in digital PCR (Fig. 2B). By contrast, digital PCR did not succeed when it was prepared by HotShot only, without subsequent desalting dialysis (data not shown).

The tumor cells S462 are known to have a heterozygotic deletion of the NF1 gene ${ }^{4}$. Consequently, digital PCR revealed that the copy number of $N F 1$ is approximately half of that of the reference gene RPP3O in the tumor cells (Fig. 2B, upper). By contrast, both genes had two copies in the non-tumor fibroblasts (Fig. 2B, lower). After confirming this genetic difference between the tumor and non-tumor cells, we used this intrinsic difference and relative copy numbers of the two genes determined in digital PCR to calculate the proportion of tumor cells in mixed cultures. The average of the calculated proportions from 4 replicates increased constantly as the proportions of tumor cells in the set-up increased (Fig. 3).

Treatment with nilotinib, a tyrosine kinase inhibitor and anticancer reagent, led to reduced vital cells in the cultures (Fig. 4A). We then asked whether or not the drug

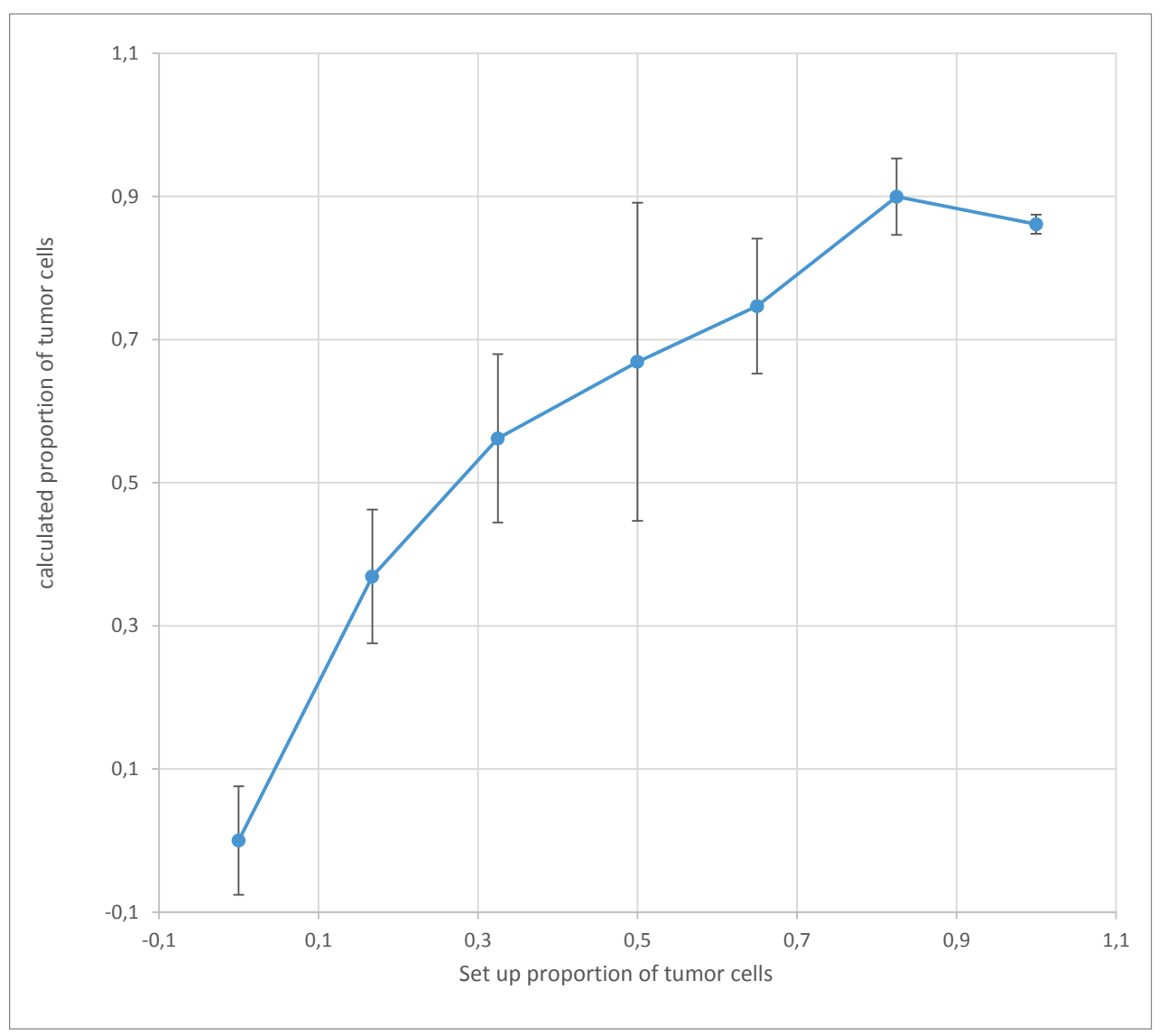

Fig. 3. Calculated proportion of tumor cells.

Tumor cells and non-tumor cells were mixed at various ratios were seeded into each well of a 96-plate. After attachment overnight, the cells were treated with HotShot/Drop-dialysis and directly subjected to digital PCR which gave the ratio of NF1:RPP30. Base on this ratio, proportion of tumor cells in each well was calculated. Mean and standard deviation of proportion of tumor cells were calculated from 4 replicates at each set-up proportion. The calculated proportions of tumor cells (Y-axis) were plotted against the set-up proportions (X-axis). 


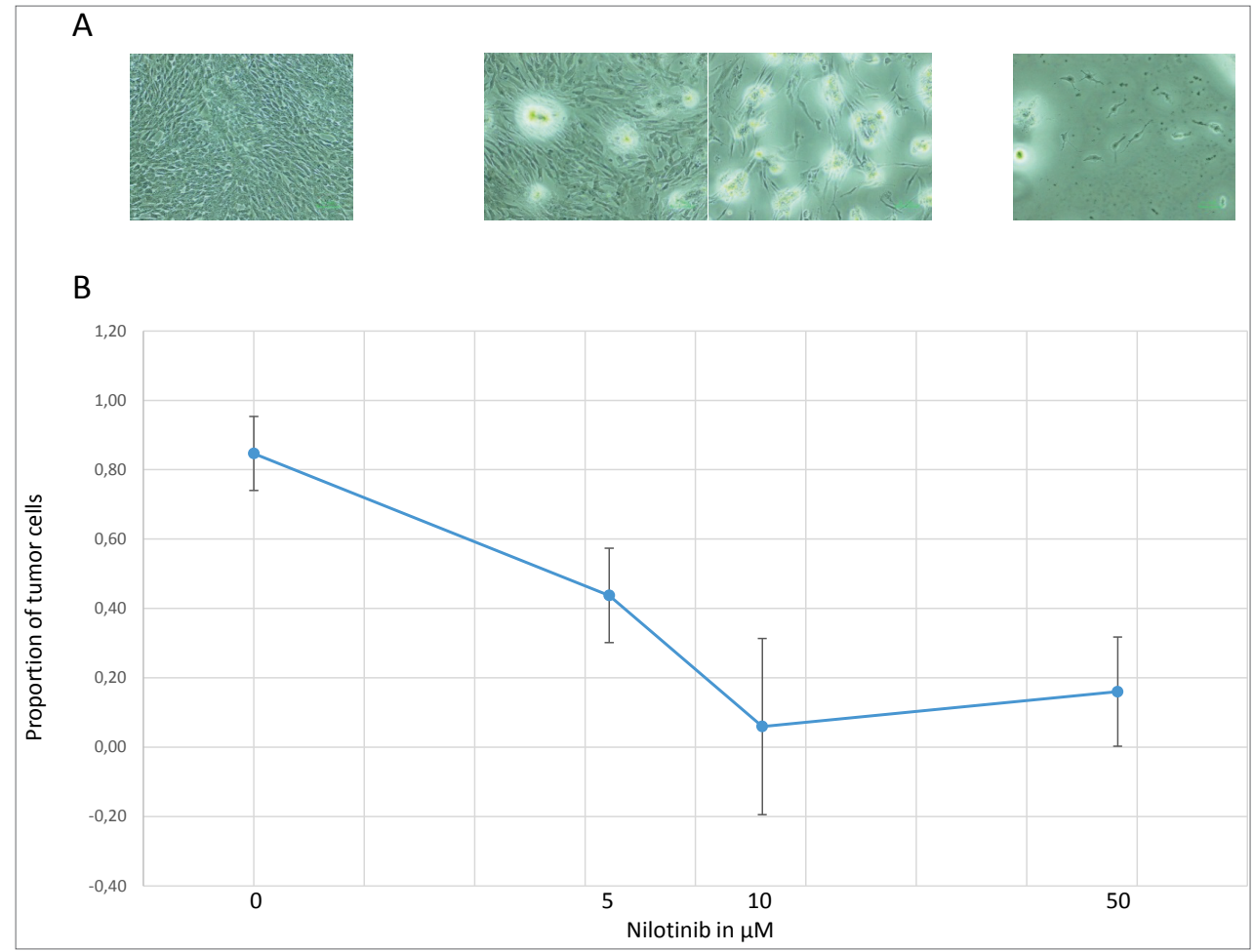

Fig. 4. Results of treatment with nilotinib for 5 days.

Vital cells were visually reduced in a dose-dependent manner (A). Proportion of tumor cells, as calculated from the ratio of NF1: RPP30, decreased in the range of 0 to $10 \mu \mathrm{M}$ and then slightly increased at the highest dose of $50 \mu \mathrm{M}$ (B).

affected tumor cells and non-tumor cells differently, in other words, whether or not the proportion of the tumor cells changed dose-dependently. Based on the ratios of NF1/RPP30 from digital PCR using DNA prepared from the treated cultures, the proportions of tumor cells were calculated which decreases as the drug-dose increases in the dose range of $0-10 \mu \mathrm{M}$ (Fig. $4 \mathrm{~B}$ ). At $50 \mu \mathrm{M}$, the highest dose tested, the proportion of tumor cells increased slightly. However, as can be seen in the above corresponding image in Fig. 4A, most cells were killed at this high concentration which is likely beyond the range of the therapeutic index (blue area in Fig. 1).

\section{DISCUSSION}

In this pilot study, we demonstrated the feasibility of our novel genetic- and cell-based strategy for assessing drug efficacy and toxicity in vitro. We showed that it is possible to calculate the proportion of tumor cells in a mixed culture using the relative quantity a tumor-specific genetic feature normalized against a universal genetic feature. In a mixed culture treated with a test drug, nilotinib, the proportion of tumor cells decreased as the dose of the test drug increased from 0 to $10 \mu \mathrm{M}$, likely indicating that the test drug is more effective on the tumor cells than on the non-tumor cells in this dose-range. The highest dose of $50 \mu \mathrm{M}$ is likely outside of the therapeutic index (Fig. 1) where both tumor cells and non-tumor cells were severely affected, as can indeed be seen in the above image (Fig. 4A).
The key concept of our strategy is to determine the proportion of tumor cells using tumor-specific genetic features. Conventionally, phenotypic markers, such as surface antigens, are more frequently used to mark, to identify, to isolate or/and to quantify tumor cells. However, most of such phenotype-based markers lack sufficient specificity, vary in expression and are difficult to quantify. By contrast, genetic markers are highly specific, stable and easy to quantify. For example, a tumor-specific mutation or copy number variation is present exclusively in the tumor cells but not in any non-tumor cells also within the same individual. Such genetic features can be reliably and precisely quantified, for example, by means of massive parallel sequencing or digital PCR. Digital PCR is a highly precise, sensitive and fast technology, which requires only a small sample amount. In a mixed culture containing tumor and non-tumor cells, the ratio of a tumor-specific heterozygously deleted sequence to a universal one varies only between 0.5 and 1 . Conventional real-time PCR therefore does not have sufficient resolution. Even with digital PCR, the deviation is still large in this pilot study. However, by optimizing the whole procedure, we expect to further reduce the deviation.

High purity DNA is not essential for digital PCR. Our samples were simply prepared by lysing the cells and desalting the lysis subsequently. Despite the rather crude nature of the DNA-containing solution, both conventional PCR (data not shown) and digital PCR were successful. This method it is simple and quick and requires no special instruments. It can therefore be carried out in any standard laboratory. Though we only tried conventional 
and digital PCRs, DNA prepared using our method may also be suitable for other downstream analysis and applications.

In this pilot study, we used the heterozygous deletion of the NFl gene as the tumor-specific genetic feature for the relative quantification of tumor cells. Based on the same principle, deletions of other genes or loci, heterozygous or homozygous, as well as mutations can also be used as tumor-specific genetic features. However, for using a mutation, the digital PCR assays for the mutant and the normal alleles need to be extensively validated to ensure their specificity for the respective allele.

Another important potential application field of this genetic- and cell-based assessment is personalized cancer care. Drugs can be tested for their efficacy on tumor cells and toxicity on non-tumor cells using primary cultures derived from resected tumors. Such a preclinical test would provide a new perspective for selecting the most suitable chemotherapeutic for each tumor, in analogue to an antibiotic-screening for infectious diseases.

Despite all these advantages and promising application potential of our genetic- and cell-based in vitro tool, we have to be aware of its limitations and in vitro nature. For example, the therapeutic index of a drug obtained using this in vitro tool may not due to the neoplastic nature of the tumor cells, but rather corresponds to the different response of different types of cells (fibroblasts vs. Schwann cells) or their different origins (from different individuals). The stronger effect on tumor cells could also be explained by faster growth of tumor cells in comparison to non-tumor cells. Most importantly, cells in vitro behavior differs from the behavior of cells in the human body and consequently, the results of in vitro assessment often do not or only partially reflect the real clinical situation. Therefore, the efficacy and toxicity of drugs obtained for cultured cells are rather of biomarker nature but cannot be considered as reliable as preclinically measured efficacy of antibiotics. Nevertheless, using mixed cultures and primary cultures is a progress from using cell lines. With extensive efforts, we are confident to find in vitro conditions under which reasonable correlation of in vitro data with real responses of patients can be obtained.

\section{CONCLUSION}

We demonstrated the feasibility of a genetic- and cellbased in vitro tool for testing the efficacy and toxicity of anticancer drugs. Additional efforts and comprehensive studies are needed for further development and optimization of this tool, which will likely have high application potential in drug discovery and personalized cancer care.

Acknowledgement: We thank Mr. Horath for his technical assistance. We also thank Dr. Volz and the research group of Dr. Dandri-Peterson for providing us the access to their digital PCR device.

Author contributions: LK: study design, methods, experiment organization and supervision, data evaluation, drafting the manuscript; $\mathrm{HH}$ : results evaluation and interpretation, critical editing the manuscript, manuscript finalization; WJ: prepared the mixed culture and carried out the treatment; IA: carried out HotShot/Drop-Dialysis and digital PCR.

Conflict of interest statement: The procedure is patentprotected (10 2013221 452, Germany).

\section{REFERENCES}

1. Double JA, Bibby MC. Therapeutic index: a vital component in selection of anticancer agents for clinical trial. J Natl Cancer Inst 1989;81(13):988-94.

2. McMillin DW, Negri JM, Mitsiades CS. The role of tumour-stromal interactions in modifying drug response: challenges and opportunities. Nat Rev Drug Discov 2013;12(3):217-28.

3. Caponigro G, Sellers WR. Advances in the preclinical testing of cancer therapeutic hypotheses. Nat Rev Drug Discov 2011;10(3):179-87.

4. Frahm S, Mautner VF, Brems H, Legius E, Debiec-Rychter M, Friedrich RE, Knofel WT, Peiper M, Kluwe L. Genetic and phenotypic characterization of tumor cells derived from malignant peripheral nerve sheath tumors of neurofibromatosis type 1 patients. Neurobiol Dis 2004;16(1):85-91.

5. Jiang W, Schnabel C, Spyra M, Mautner VF, Friedrich RE, Hagel C Manley PW, Kluwe L. Efficacy and selectivity of nilotinib on NF1associated tumors in vitro. J Neurooncol 2014;116(2):231-6.

6. Montero-Pau J, Gomez A, Munoz J. Application of an inexpensive and high-throughput genomic DNA extraction method for the molecular ecology of zooplanktonic diapausing eggs. Limnology and Oceanography-Methods 2008;6:218-22.

7. Saraswat M, Grand RS, Patrick WM. Desalting DNA by drop dialysis increases library size upon transformation. Biosci Biotechnol Biochem 2013;77(2):402-4 